\title{
Capítulo 7 Algoritmos Genéticos en el Problema de Picking
}

\section{Chapter 7 Genetic Algorithms in the Picking Problem}

CASARRUBIAS-VARGAS, Heriberto †**, VÁSQUEZ-GODÍNEZ, Noé \& CHAVÉZ-HERNÁNDEZ, Maribel

Universidad Autónoma del Estado de México. Centro Universitario UAEM Valle de México. Blvd. Universitario s/n Predio San Javier, Atizapán de Zaragoza, México, C.P. 54500

ID $1^{\text {er }}$ Autor: Heriberto Casarrubias-Vargas / ORC ID: 0000-0003-3429-0010, CVU CONACYT ID: 167638

ID $1^{\text {er }}$ Coautor: Noé Vásquez-Godínez / ORC ID: 0000-0001-8678-1001

ID $2^{\text {do }}$ Coautor: Maribel Chávez-Hernández, ORC ID: 0000-0001-9349-8044

DOI: $10.35429 / \mathrm{H} .2020 .1 .98 .116$

H. Casarrubias, N. Vásquez y M. Chavéz

G. Gaviño \& R. Pérez. (AA.VV). Políticas, Casos de estudio, Técnicas de simulación y Programas de competencias en la educación de logística y cadena de suministro en México. Handbooks-@ECORFAN-Mexico, Estado de México. 2020. 


\section{Resumen}

El problema de picking forma parte del problema de recolección de ítems en un almacén para un pedido dado y es el más costoso del proceso; optimizar el tiempo en la recolección de ítems es importante debido a que es un cuello de botella en la tasa de servicio de ordenes atendidas y tiene un impacto significativo en la eficiencia de la cadena de suministro. En este capítulo se propone una estrategia para reducir el tiempo de recolección minimizando la trayectoria de recolección de ítems. El algoritmo usa una optimización mediante algoritmos genéticos y se realiza una comparativa con otras estrategias heurísticas en almacenes con distintas morfologías y considerando ordenes de distintos tamaños.

\section{Algoritmos Genéticos, Problema de Picking, Optimización de Tiempo de Recorrido, Algoritmos Heurísticos}

\section{Abstract}

The picking problem is part of the item picking problem in a warehouse for a given order and is the most expensive in the process; Optimizing the item collection time is essential because it is a bottleneck in the service rate of orders fulfilled and has a significant impact on the efficiency of the supply chain. In this chapter, is proposed a strategy to reduce the picking time by minimizing the path. The algorithm uses a genetic algorithm by optimizing, and a comparison is performed with other heuristic strategies in warehouses with different morphologies and considering orders of different sizes.

\section{Genetic Algorithms, Picking Problem, Travel Time Optimization, Heuristic algorithms}

\section{Introducción}

Los almacenes son un componente esencial para la gestión de cualquier operación logística y gestión de la cadena de suministro, el almacenamiento controla el movimiento, envió, recepción y colecta de mercadería; debido a que es el componente responsable de vincular proveedores, sistemas de producción, sistemas de distribución y puntos de venta; el desempeño de los almacenes afecta significativamente la cadena de suministro (Roodbergen, Iris, \& Taylor, 2015) por lo cual es importante establecer políticas sobre la operación del almacén que mejoren su eficiencia.

En los almacenes se pueden categorizar las distintas actividades que se realizan en su operación y se distinguen en: almacenamiento, recepción de mercaderías, recepción de pedidos, preparación de pedidos, clasificación, embalaje y envío. Cada una de estas actividades son dependientes del contexto y del diseño del almacén.

En los almacenes se debe de recolectar artículos o ítems que se encuentran distribuidos espacialmente en el almacén, un sistema bastante popular es el denominado picker to parts en donde se debe recorrer los pasillos del almacén para recuperar los productos almacenados en estantes para satisfacer el pedido de un cliente (Bozer \& Kile, 2008) cada articulo se puede encontrar distribuido en diferentes locaciones del almacén lo cual implica un desplazamiento y una planeación para su recolección.

Dentro de las distintas actividades a realizar en un almacén el proceso de picking o de recolección de ítems es el más laborioso y repetitivo generando desde $50 \%$ a $70 \%$ de los costos operativos del almacén (Bartholdi \& Hackman, 2014). El tiempo de proceso de picking se descompone en tiempo de desplazamiento $50 \%$, tiempo de búsqueda $20 \%$, tiempo de recolección $15 \%$, tiempo de preparación $10 \%$ y otros 5\% (Henn, Koch, \& Wäscher, 2012).

\section{Modelo matemático para optimización de picking}

El problema de picking puede ser abordado de forma analítica mediante un modelo de programación entera para conseguir la solución óptima al problema. En el trabajo de (Valle, Beasley, \& da Cunha, 2016) se plantea un modelo que emplea el uso de variables de decisión binaria, para indicar cuando debe tomarse la carga. 
En el modelo de Valle para la solución se expone en dos fases, calcular la ruta más corta entre dos ubicaciones en un almacén y como segunda fase se enfoca en el problema de optimización de encontrar la mejor secuencia de selección para minimizar las distancias recorridas, esto se asemeja a problemas de optimización combinatoria, estos problemas exponen niveles de complejidad altos.

El tiempo requerido que emplean las soluciones matemáticas para encontrar la estrategia de recolección de ítems debe de ser minimizado para cumplir de forma práctica con los pedidos; ya que la operación de surtir las ordenes de pedido afectan directamente el tiempo de finalización del pedido impactando en el servicio a clientes.

La complejidad de un algoritmo se divide en dos clases, una de ellas son de tipo $\mathrm{P}$ cuyos algoritmos de solución son de complejidad polinomial en tiempo; y la otra clasificación son los problemas NP, cuya solución hasta la fecha no han podido ser resueltos de manera exacta por medio de algoritmos deterministas eficientes pero que pueden ser resueltos por algoritmos no deterministas o heurísticos (Garey \& Jhonson, 1979). Los problemas de picking son de tipo NP-Hard, en los cuales para su solución se emplean heurísticas y meta heurísticas; considerar obtener la solución óptima de recorrido para preparar cada orden se vuelve impráctico debido al coste computacional, tiempo requerido y volumen de ordenes en un periodo de tiempo.

\section{Estrategias de recolección heurísticas}

Considerando el impacto del costo en tiempo de desplazamiento en la operación de un almacén y el costo de encontrar la solución óptima se han desarrollado técnicas heurísticas que permiten organizar el recorrido o colecta de ítems de una orden que ayudan a disminuir el tiempo de recolección (Scholz, Schubert, \& Wäsher, 2017). Cada una de estas estrategias intuitivas tienen distinto coste en tiempo de recorrido y en general no obtienen el tiempo óptimo. Sin embargo, son muy usuales en la práctica debido al bajo costo de implementación y tiempo requerido para la planificación.

\section{Return}

En esta estrategia, por cada recolección se entra a los pasillos, no se cubre todo el pasillo, solo hasta donde se encuentre el ultimo articulo por recolectar, una vez que se recolecta se regresa hasta el inicio de los pasillos y procede con el resto.

Figura 7.1 Estrategia de recolección Return

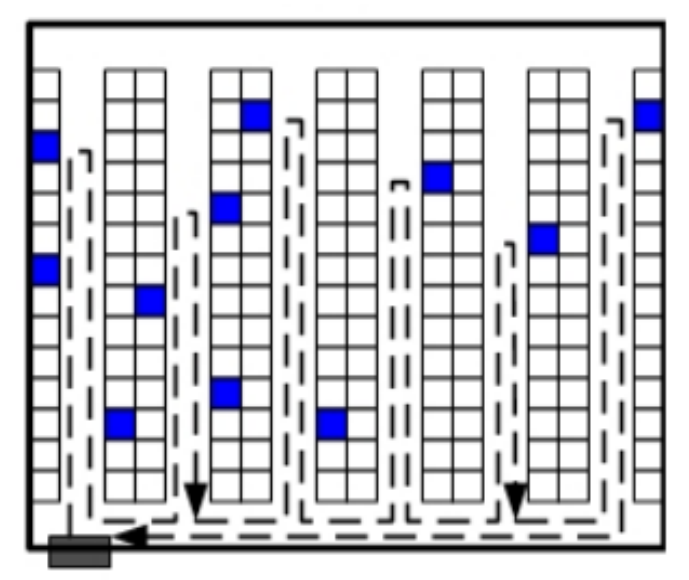

\section{S-Shape}

La estrategia en forma de $\mathrm{S}$ también se llama estrategia transversal conduce a una ruta en la que los pasillos, que deben ser visitados se recorren completamente. Se omiten los pasillos donde no hay nada que recoger. Por lo tanto, los pasillos se visitan en forma de S. El recolector ingresa así a un pasillo desde un extremo y sale del pasillo desde el otro extremo, comenzando en el lado izquierdo del almacén. Después de elegir el último artículo, el preparador de pedidos regresa al frente del pasillo. Esta estrategia se usa con frecuencia, porque es muy simple de usar y entender. 
Figura 7.2 Estrategía de recolección S-shape

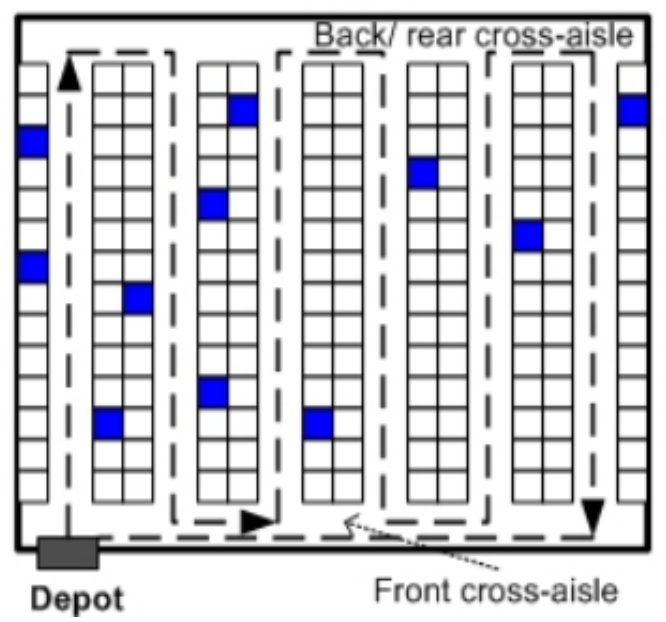

\section{Largest Gap}

En la estrategia Largest Gap se recorren completamente el primer y último pasillo que contengan ítems a recolectar. El recolector recorre los otros pasillos sin atravesar el mayor espacio entre cada par de ítems vecinos a recoger dentro del mismo pasillo, a este espacio se le conoce como el largest gap. La heurística (Largest Gap) es especialmente útil cuando el tiempo para cambiar pasillos es corto y el número de selecciones por pasillo es bajo.

Figura 7.3 Estrategía de recolección Largest Gap

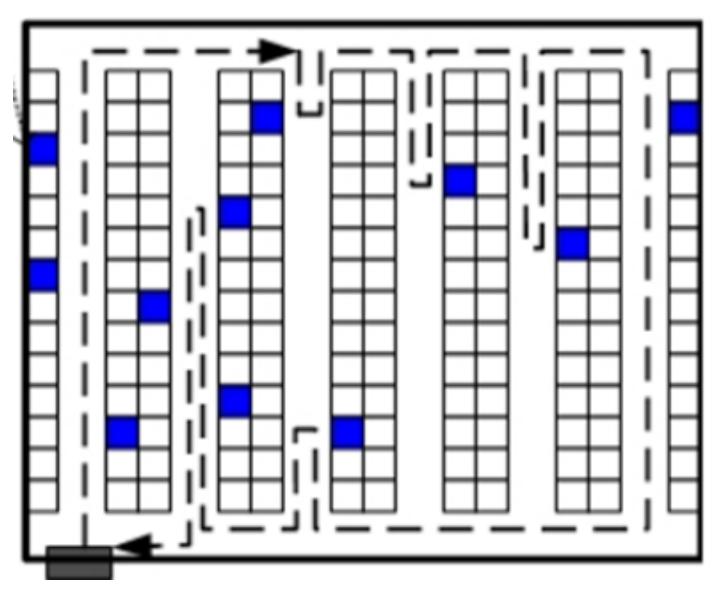

\section{Layout del almacén}

La organización y distribución del espacio en distintas áreas operativas comprende al diseño de almacenes, de forma general un almacén cuenta con: recepción, control de calidad, adaptación de unidades de carga, almacenamiento, preparación de pedidos y despachos. Una elección adecuada de la distribución del área de almacenamiento incide en el performance de las operaciones (Jinxiang, Goetschalckx, \& McGinnis, 2009) en general la elección de la distribución del almacén depende de la operación en particular.

Para el problema de picking el área relevante es la zona de almacenamiento; que en general se considera como un arreglo rectangular que contiene los racks, lugares en donde se almacenan los ítems, y los pasillos por donde se puede transitar para realizar la recolección de ítems. Las ubicaciones de almacenamiento son de tamaño idéntico y dispuesto a ambos lados de los pasillos; por los cuales los preparadores de pedidos están habilitados para entrar y salir para colectar los ítems. La parte del almacén entre dos pasillos transversales adyacentes se denomina bloque y la parte correspondiente del pasillo por donde se puede transitar se denota como un sub aislé o pasillo. A partir de este escenario se analizan diversas estrategias de solución al problema de picking 
En el presente estudio sin pérdida de generalidad se considera un diseño de estantes con capacidades de almacenamiento iguales entre ellas y con una distribución de forma paralela entre si, para formar un bloque almacén; se considera el punto de despacho en la parte superior izquierda en la cual se da inicio y fin a la recolección de los diferentes ítems dentro del almacén. En el ejemplo de la figura 7.4 el diseño del almacén tiene 4 pasillos verticales y 3 horizontales.

Figura 7.4 Layout de almacén, con un triangulo invertido se marca el punto de despacho

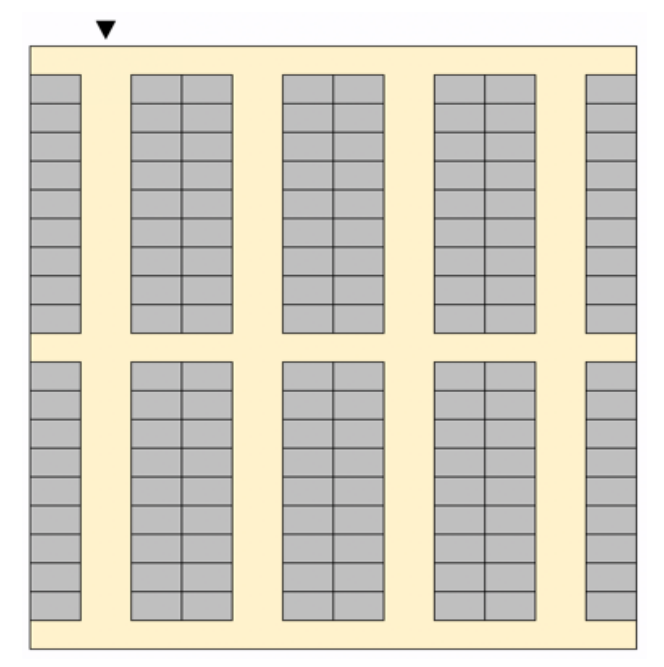

Los parámetros que definen a un layout son:

1. Número de racks, el conjunto de celdas contiguas que almacenan los ítems.

2. Número de celdas en cada rack.

3. Número de pasillos verticales.

4. Pasillo frontal y pasillo del fondo, primer y último pasillo horizontal; para un layout dado se considera que al menos existen este par de pasillos.

5. Número de pasillos horizontales, al menos son dos para todo layout.

Para realizar una comparativa en el rendimiento de las estrategias de recolección de ítems se considera una distribución aleatoria uniforme para la ubicación de los ítems y un conjunto de layouts con los siguientes parámetros:

1. Número de pasillos horizontales: $3,4,5, \ldots, 10$

2. Número de pasillos verticales: $3,4,5, \ldots, 10$

3. Número de ítems por orden: $5,10, \ldots, 50$

El número total de experimentos por combinación de parámetros, pasillos horizontales, pasillos verticales y cantidad de ítems por orden, es establecido a 20; para un total de 6400 experimentos, por cada experimento se evalúan cuatro algoritmos de recorrido: S-shape, Return, Largest Gap y un enfoque de solución basado en un algoritmo genético que se describe a continuación.

\section{Estrategia de recolección basada en algoritmos genéticos}

Los algoritmos genéticos usan una estrategia basada en una búsqueda heurística dirigida en el espacio de posibles soluciones, se encuentran inspirados en el fenómeno de la evolución biológica, propuesta original de John Henry Holland (Holland, 1992).

Para operar con los algoritmos genéticos se requieren de varios elementos; primero la codificación del problema en una representación particular denominada fenotipo que contiene la semántica de la solución al problema planteado; es necesario de un conjunto de individuos o soluciones denominado población, también se requiere la validación del individuo como solución del problema y operadores sobre las soluciones o individuos, operador de mutación y operador de cruzamiento que trabajan sobre el espacio de soluciones para generar nuevos individuos con potencialmente mejor rendimiento. 
El funcionamiento del algoritmo genético se representa gráficamente en la figura 7.5 en donde se observan los distintos componentes: conjunto de soluciones iniciales, función objetivo, operadores para generar nuevas soluciones tomando como base las soluciones actuales y un criterio de paro o cuando dejar de buscar la siguiente mejor solución.

Figura 7.5 Algoritmo genético i: inicialización, $\mathrm{f}(\mathrm{X})$ : evaluación, ?: condición de término, Se: selección, Cr: cruzamiento, Mu: mutación, Re: reemplazo, X*: mejor solución. (Nojhan, 2007)

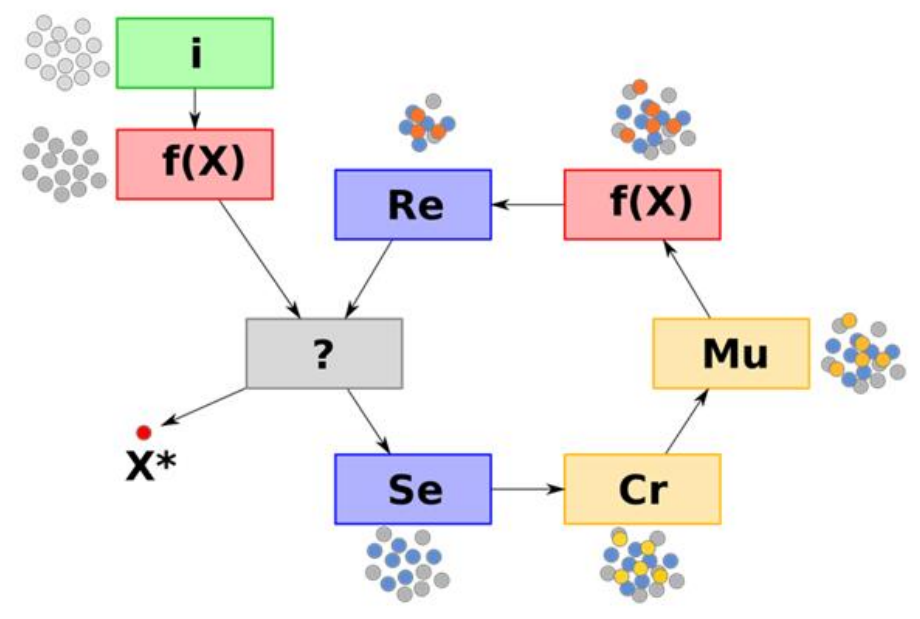

En esencia el algoritmo genético es un procedimiento de optimización iterativo que parte de un conjunto de soluciones admisibles, denominada población inicial; para el problema de picking una solución admisible es el orden de recolección de ítems en el almacén que es codificado como un vector con entradas numéricas al que se le denomina individuo o fenotipo.

Posteriormente ordena las soluciones de acuerdo con la eficiencia de la solución, que se le llama aptitud o calidad del individuo. En base a la calidad de las soluciones construye un conjunto de nuevas soluciones, siguiente generación, tomando en consideración las mejores características de los individuos de la población. En algoritmos genéticos la evolución o herencia de características entre generaciones es un componente fundamental y emulado mediante un operador binario llamado cruzamiento y un operador unario denominado mutación. En las siguientes subsecciones detallaremos la forma de modelar el problema de picking en almacenes

\section{Codificación del problema de recorrido como fenotipo}

El algoritmo genético requiere de una codificación del problema en un vector de características regularmente con entradas que toman valores enteros, las cuales definen una solución para el problema de recorrido del almacén en la colecta de ítems de una orden o pedido.

Por consideraciones de complejidad en el tamaño del problema y basados en las heurísticas que se usan para establecer estrategias de recorrido (S-shape, Return, Largest-Gap) se realiza un particionamiento del área del almacén. 
Figura 7.6 Particionado del almacén, el punto de despacho se marca con un triángulo invertido

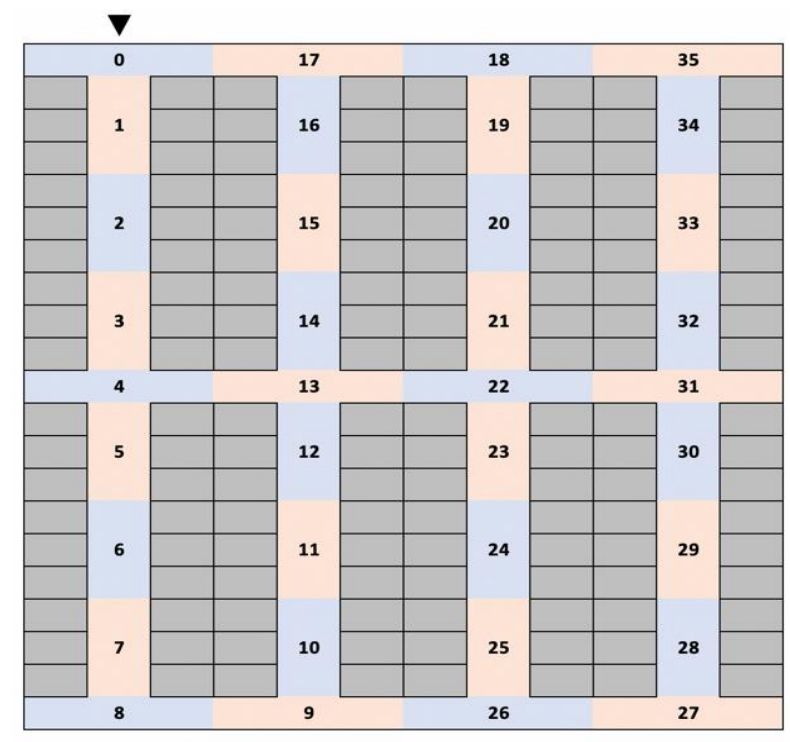

Para un conjunto dado $\mathrm{X}$, una partición de $\mathrm{X}$, denotada por $\mathrm{P}(\mathrm{X})$ es una familia de subconjuntos $\mathrm{Xi}$ con las siguientes propiedades:

$$
\begin{aligned}
& X i=\varnothing \\
& \text { Ui Xi }=X
\end{aligned}
$$

En el particionamiento del almacén se tomarán los elementos Xi del mismo tamaño para obtener una la longitud homogéna de recorrido sobre cada elemento de la partición.

La partición del área del almacén se establece de la siguiente forma, para cada bloque de racks se segmenta el pasillo vertical es tres zonas de la misma longitud y para los pasillos horizontales del almacén se le asocia un área que cubre de la mitad del bloque anterior a la mitad del bloque siguiente, como se muestra en la figura 7.6 El particionamiento se aplica para generar un nivel de discretización sobre el almacén y permite reducir el tiempo de búsqueda de recorrido, lo anterior debido a que considera un espacio menor en un factor de 1:3 en los racks.

Note que el particionamiento en tres regiones es independiente de la longitud del rack, para el caso de racks de longitud mayor a nueve el particionamiento del rack se sigue realizando en tres regiones, dos contiguas a los pasillos horizontales y una central. Para almacenes con más racks no se cambia el nivel de particionamiento, sin embargo, se contemplan más particiones sobre el layout del almacén.

Para la búsqueda de rutas adecuadas de recolección; se aplica una estrategia en dos pasos, primero se determinan las zonas de particionamiento a considerar seguido de una optimización de la ruta para el recorrido de las zonas de particionamiento.

Dada una orden $\mathrm{O}=\left\{\right.$ item $_{1}$, item $_{2}, \ldots$, item $\left._{\mathrm{n}}\right\}$ compuesta de $\mathrm{n}$-ítems, la ruta a seguir se codifica en términos del particionado y el ordenamiento en que se deben visitar para realizar la recolección. Si denotamos que el ítem $\mathrm{i}_{\mathrm{i}}$ se encuentra en el bloque $\mathrm{B}_{\mathrm{k}(\mathrm{i})}$; una sucesión u orden para visitar los bloques en el almacén se puede codificar mediante el siguiente vector:

$$
\left[\begin{array}{lllll}
\mathrm{B}_{1} & \mathrm{~B}_{\mathrm{k} 2} & \mathrm{~B}_{\mathrm{k} 3} & \mathrm{~B}_{\mathrm{k} 4} & \mathrm{~B}_{1}
\end{array}\right]
$$

Obsérvese que todos los recorridos empiezan y finalizan en el bloque 1, el punto de despacho. El orden de los bloques en el vector se interpreta como una prioridad sobre que bloque es más importante visitar primero, lo cual implícitamente establece el orden de recolección de ítems.

Por ejemplo, considere los ítems en la orden dada por: $\mathrm{O}=\left\{\right.$ item $_{1}$, item $_{2}, \ldots$, item 8$\}$ distribuidos espacialmente en los racks y zonas de particionamiento como se muestra en la figura 7.7. 
El conjunto de bloques de particionamiento a considerar en la codificación del problema de recorrido como un individuo para el algoritmo genético considera las particiones:

$$
\left\{\mathrm{P}_{1}, \mathrm{P}_{5}, \mathrm{P}_{19}, \mathrm{P}_{25}, \mathrm{P}_{33}, \mathrm{P}_{32}\right\}
$$

Una codificación como solución valida para realizar el recorrido y colecta de ítems esta dada por la visita de los siguientes bloques:

$$
\begin{aligned}
& \mathrm{P}_{1} \rightarrow \mathrm{P}_{2} \rightarrow \mathrm{P}_{3} \rightarrow \mathrm{P}_{4} \rightarrow \mathrm{P}_{5} \rightarrow \mathrm{P}_{6} \rightarrow \mathrm{P}_{7} \rightarrow \mathrm{P}_{8} \rightarrow \mathrm{P}_{26} \rightarrow \mathrm{P}_{25} \rightarrow \mathrm{P}_{24} \rightarrow \mathrm{P}_{23} \rightarrow \mathrm{P}_{22} \rightarrow \mathrm{P}_{21} \rightarrow \mathrm{P}_{20} \rightarrow \mathrm{P}_{19} \rightarrow \mathrm{P}_{18} \rightarrow \mathrm{P}_{35} \\
& \rightarrow \mathrm{P}_{34} \rightarrow \mathrm{P}_{33} \rightarrow \mathrm{P}_{32} \rightarrow \mathrm{P}_{31} \rightarrow \mathrm{P}_{22} \rightarrow \mathrm{P}_{13} \rightarrow \mathrm{P}_{4} \rightarrow \mathrm{P}_{3} \rightarrow \mathrm{P}_{2} \rightarrow \mathrm{P}_{1}
\end{aligned}
$$

Figura 7.7 Ejemplo de una orden de ítems distribuidas en el layout del almacén

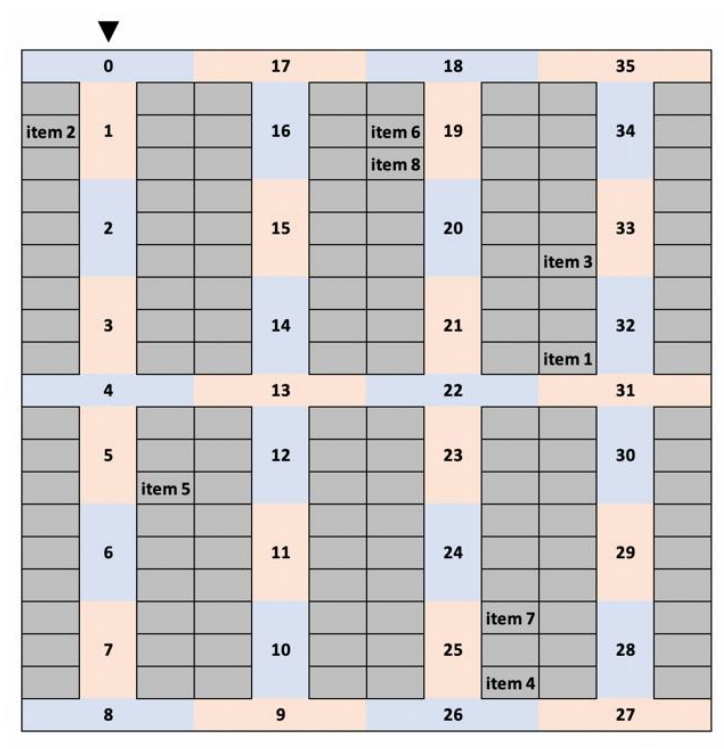

Considerando el layout planteado se observa que los desplazamientos permisibles dada una posición en un bloque son movimientos en horizontal o en vertical, un mundo tipo manhattan, la representación anterior se puede simplificar considerando únicamente los puntos de cambio de dirección en la trayectoria, con lo cual la sucesión anterior puede ser equivalentemente denotada por:

$$
\mathrm{P}_{1} \rightarrow \mathrm{P}_{8} \rightarrow \mathrm{P}_{26} \rightarrow \mathrm{P}_{18} \rightarrow \mathrm{P}_{35} \rightarrow \mathrm{P}_{31} \rightarrow \mathrm{P}_{4} \rightarrow \mathrm{P}_{1}
$$

La trayectoria anterior tiene una longitud de 76 y visita los bloques que contienen a los ítems en el siguiente orden:

$$
\left[\mathrm{P}_{1}, \mathrm{P}_{5}, \mathrm{P}_{25}, \mathrm{P}_{19}, \mathrm{P}_{33}, \mathrm{P}_{32}\right]
$$

Otro posible recorrido en el almacén puede ser establecido como:

$$
\mathrm{P}_{1} \rightarrow \mathrm{P}_{8} \rightarrow \mathrm{P}_{26} \rightarrow \mathrm{P}_{25} \rightarrow \mathrm{P}_{26} \rightarrow \mathrm{P}_{27} \rightarrow \mathrm{P}_{35} \rightarrow \mathrm{P}_{18} \rightarrow \mathrm{P}_{19} \rightarrow \mathrm{P}_{18} \rightarrow \mathrm{P}_{1}
$$

Teniendo una longitud de 66 y el orden de visita de los bloques conteniendo los ítems es:

$$
\left[\mathrm{P}_{1}, \mathrm{P}_{5}, \mathrm{P}_{25}, \mathrm{P}_{32}, \mathrm{P}_{33}, \mathrm{P}_{19}\right]
$$

Obsérvese de las trayectorias anteriores:

1. Los recorridos tienen longitud variable para una misma lista de bloques a visitar o equivalentemente ítems a colectar.

2. La longitud del recorrido es calculable a partir de la representación en fenotipos.

3. La longitud del recorrido es calculable a partir de la representación en particiones.

4. Con esta codificación pueden ser representadas las heurísticas tradicionales: Return, S-shape, Largest Gap y demás. 
Codificar en el algoritmo genético un fenotipo de longitud variable computacionalmente es costoso, por lo cual se representa el fenotipo por el orden de visita de los bloques para obtener representaciones de longitud fija; los individuos son permutaciones sobre la prioridad de visita en los bloques que contienen los ítems a colectar; este enfoque no especifica la ruta a seguir de un elemento de la partición a otro, no describe la lista de las particiones intermedias a transitar. Sin embargo, considerando que se busca minimizar la longitud de la trayectoria, se puede usar la ruta más corta entre pares de bloques.

La distancia mínima entre pares de bloques puede ser pre-calculada usando algebra matricial, basados en la matriz de incidencia asociada al layout del almacén, la cual se define de la siguiente forma:

La matriz de adyacencia asociada a los bloques del layout en el almacén es una matriz cuadrada, $\mathrm{A}(\mathrm{aij})$, de dimensión n, siendo n el número de bloques en la partición del layout, en donde la entrada aij de la matriz A se define como 1 si el elemento i de la partición es contiguo al elemento j de la partición. Por ejemplo, en el layout de la figua 7.4 el elemento $\mathrm{P}_{0}$ de la partición es contiguo a los elementos $\mathrm{P}_{17}$ y $\mathrm{P}_{1}$ pero no es contiguo al elemento $\mathrm{P}_{2}$.

Las potencias de la matriz de adyacencia del layout correspondiente al almacén contienen información acerca de las trayectorias entre los bloques de la partición. Para una potencia k de la matriz de adyacencia A, denotada por:

$$
P\left(p_{i j}\right):=A^{k}=\underbrace{A \times A \times \ldots A}_{k \text {-veces }}
$$

El valor que toma la entrada $\mathrm{p}_{\mathrm{ij}}$ en la matriz $\mathrm{P}$ toma el valor del número de trayectorias que existen de longitud $k$ entre los elementos de la partición Pi y $P_{j}$. En base a esta propiedad (Korte \& Vygen, 2010) puede determinarse la longitud de la trayectoria mínima entre pares de bloques.

Figura 7.8 Izquierda, visualización de la matriz de adyacencias para el layout en la Figura 7.6. A la derecha visualización de distancias minimas entre elementos de la partición. En los bordes se muestra el número de partición
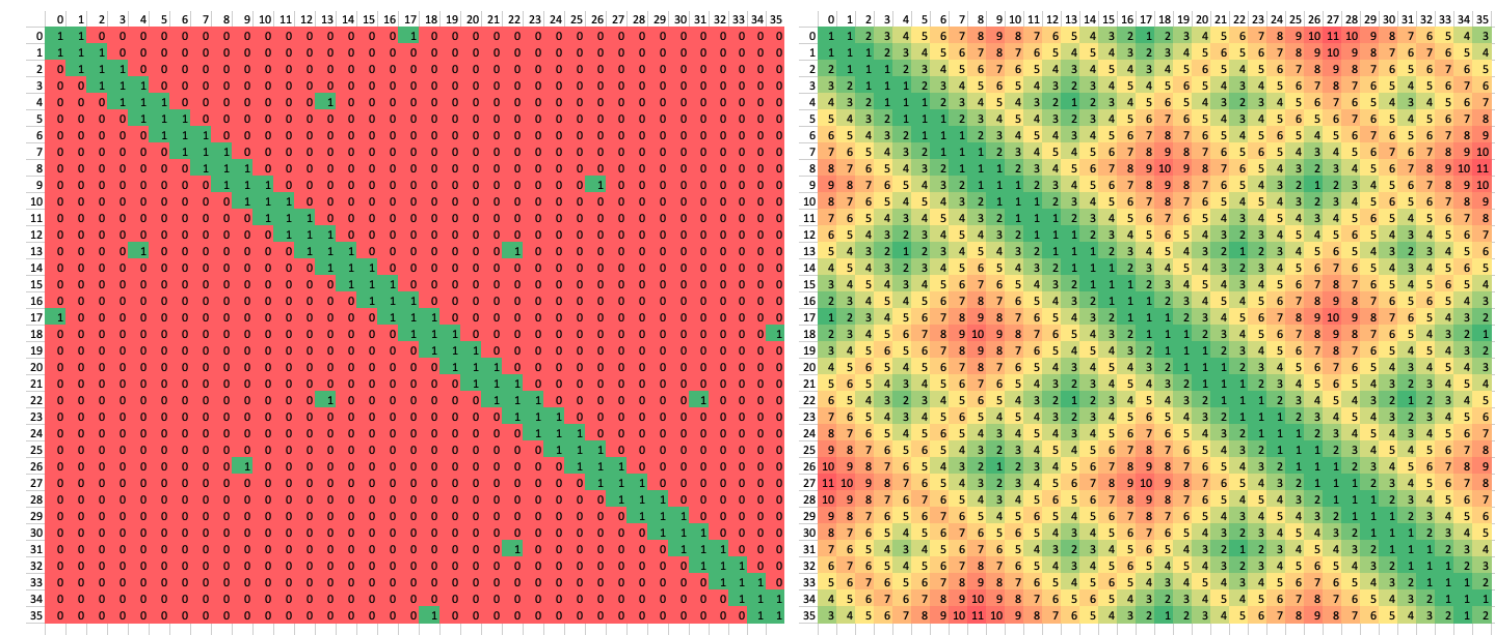

Usando la matriz de potencias correspondiente a la matriz de adyacencias se puede construirse una matriz que contiene la trayectoria mínima entre los distintos pares de bloques (Aho, Ullman, \& Hopcroft, 1983). La potencia máxima a calcular para determinar la longitud de la trayectoria mínima se corresponde con la cardinalidad del conjunto de bloques en el particionamiento del layout. 
Figura 7.9 Calculo de la matriz de distancias mínima

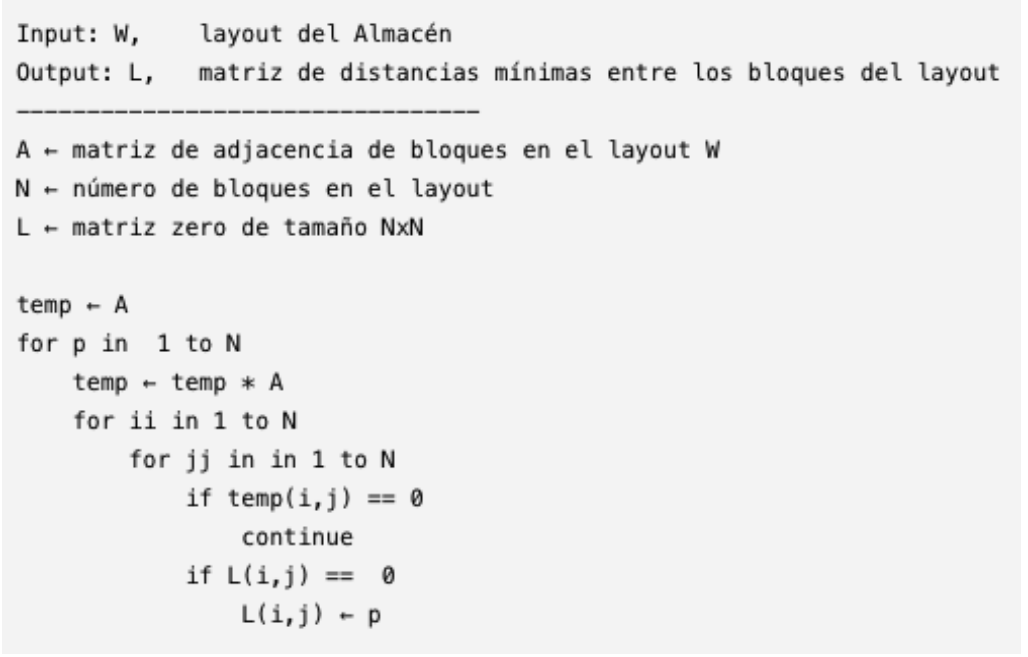

Para el caso del ejemplo dado en la Figura 7.8 la potencia máxima es 35, debido a que existen 35 bloques en el particionamiento. Esta matriz de distancias mínima puede ser pre-calculada usando la Figura 7.9 y sirve para cualquier orden de ítems que se requiera optimizar.

En la figura 7.8 se muestra la matriz de adyacencias y la de longitudes de trayectorias mínimas entre elementos de la partición, los valores de las distancias se visualizan como un mapa de calor; note los patrones que se forman debido a la distribución espacial del layout.

La matriz de distancia mínima entre bloques proporciona la longitud de trayectoria mínima sin embargo no proporciona la sucesión de bloques que componen la trayectoria de longitud mínima. Para encontrar la trayectoria de longitud mínima, ver Figura 7.24 entre pares de elementos Pi y Pj se tienen distintos escenarios dependiendo de la posición de los elementos de partición $\mathrm{Pi}$ y $\mathrm{Pj}$ en el layout del almacén, los cuales enumeramos a continuación:

1. Los bloques se encuentran en el mismo pasillo vertical

2. Los bloques se encuentran entre los mismos pasillos horizontales, están en el mismo renglón de racks horizontales

3. Los bloques se encuentran en distintos renglones de racks horizontales

Figura 7.10 Casos de distribución espacial para el cálculo de trayectoria mínima entre pares de elementos de la partición. Esquina superior izquierda caso1, esquina superior derecha caso 2, esquina inferior izquierda caso 3, esquina inferior derecha detalle de distancias caso 3

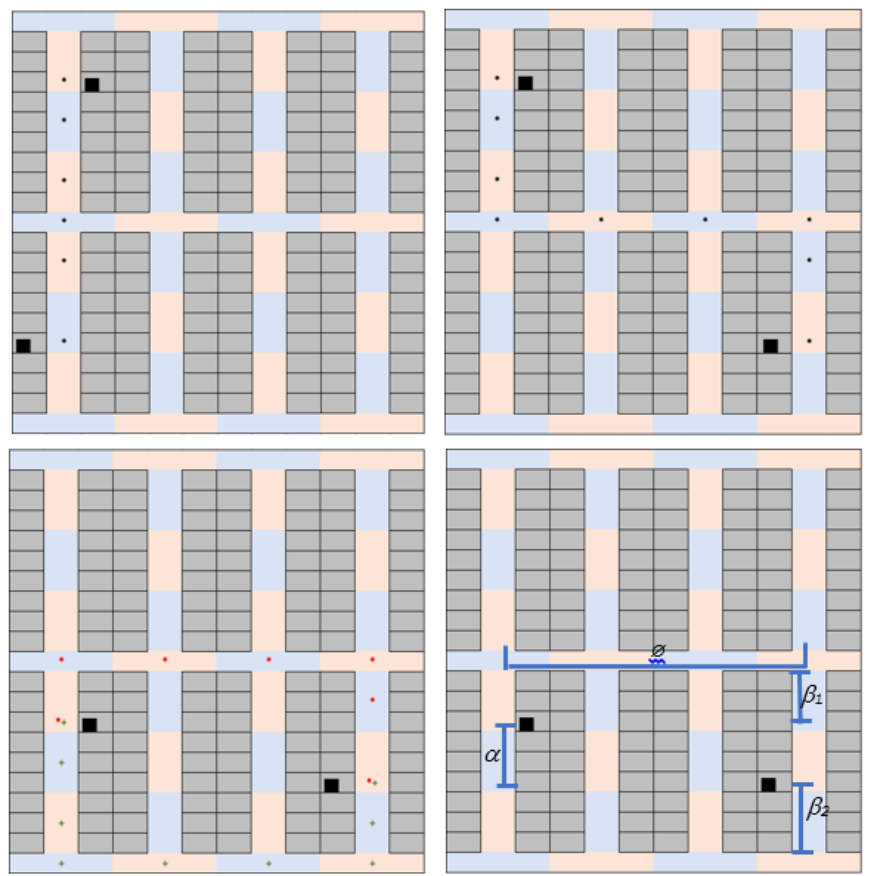


Para el primer caso la trayectoria mínima es trivial; en el segundo caso la trayectoria mínima se obtiene al recorrer en vertical hacia el primer pasillo horizontal en dirección del bloque destino, después de mueve en horizontal hacia el pasillo vertical destino y finalmente se mueve en vertical hacia el bloque destino; el recorrido usando esta política es mínima pues se asume un layout tipo manhattan.

En el tercer caso existen dos recorridos a considerar, hacer el recorrido por el pasillo horizontal de arriba o por el pasillo horizontal de abajo. La distancia es diferente dependiendo de las distancias hacia los extremos, ver Figura 7.10 imágenes del renglón inferior. La trayectoria por arriba tiene una longitud de:

$$
\beta 1+\phi+\alpha
$$

y la trayectoria por el pasillo de abajo tiene una longitud de:

$$
\alpha+\phi+\beta 2
$$

comparando ambas trayectorias en general se tiene que la trayectoria por arriba será más corta si se cumple que:

$$
\beta 1+\phi+\alpha \leq \alpha+\phi+\beta 2
$$

cancelando términos, la desigualdad anterior se mantendrá si y solo si:

\section{$\beta 1 \leq \beta 2$}

Por tanto, si $\beta 1 \leq \beta 2$ la trayectoria mínima para alcanzar a $\mathrm{Pj}$ es por el pasillo superior, en caso contrario la ruta con distancia mínima es por el pasillo de abajo.

Con lo anterior, dados dos elementos de la partición Pi y Pj en el layout de un almacén siempre se puede encontrar la ruta mínima que los conecta. Para la codificación del fenotipo en el algoritmo genético es suficiente con representar el orden de visita de los bloques y no la trayectoria completa.

El enfoque utilizado para minimizar la trayectoria total esta basado en la minimización de trayectoria entre pares de bloques, método de programación dinámica sustentado por el principio de optimalidad de Bellman (Bellman, 1957) que establece:

"Una secuencia óptima de decisiones que resuelve un problema debe cumplir la propiedad de que cualquier subsecuencia de decisiones, que tenga el mismo estado final, debe ser también óptima respecto al subproblema correspondiente"

Así para la codificación del fenotipo de una orden dada: $\mathrm{O}=\left\{\right.$ item $_{1}$, item $_{2}, \ldots$, item $\left._{n}\right\}$ primero se obtienen los bloques a visitar

$$
\{\mathrm{B} 1, \mathrm{~B} 2, \ldots, \mathrm{Bk}\}
$$

y posteriormente se representa una individuo como una permutación de los bloques a visitar:

$$
\left(\mathrm{B}_{\pi(1)}, \mathrm{B}_{\pi(2)}, \ldots, \mathrm{B}_{\pi(\mathrm{k})}\right)
$$

en donde el orden de aparición en la tupla se interpreta como el orden de visita del bloque en la colecta de ítems del almacén; al espacio de permutaciones posibles lo denotaremos por $\Omega_{\mathrm{k}}$ siendo k el número de bloques a visitar.

El espacio de búsqueda de las soluciones crece combinatoriamente en función del número de bloques a visitar dada una orden $\mathrm{O}$. Si el número de bloques a visitar es $\mathrm{N}$ el espacio de soluciones tiene tamaño $\mathrm{N}$ !, el tamaño del espacio de soluciones $\Omega_{\mathrm{k}}$ crece de forma combinatorial en relación al número de bloquea a visitar asociados a los items en una orden dada. 


\section{Operador de cruzamiento}

El algoritmo genético requiere de una operación binaria sobre el espacio de soluciones, que dadas dos soluciones codificadas como fenotipos genere una nueva solución heredando propiedades de los padres. El operador de cruzamiento, $\bowtie i$, es una operación binaria definida en los individuos de la población, $\Omega \mathrm{k}$, dependiente del punto de corte $\mathrm{i}$ :

$$
\bowtie_{\mathrm{i}:} \Omega \mathrm{k} \times \Omega \mathrm{k} \rightarrow \Omega \mathrm{k}
$$

basamos su definición en el operador de (Davis, 1985) con la variante de considerar el operador de cruza en términos de la prioridad de recorrido de los padres.

Sea una orden dada $\mathrm{O}, \mathrm{y} \mathrm{B} 1, \mathrm{~B} 2, \ldots, \mathrm{Bm}$ los bloques a visitar y sean $\mathrm{X}, \mathrm{Y}$ las soluciones dadas por:

$$
\begin{aligned}
& \mathrm{X}:(\mathrm{B} \pi \mathrm{x} 1, \mathrm{~B} \pi \mathrm{x} 2, \ldots, \mathrm{B} \pi \mathrm{xm}) \\
& \mathrm{Y}:(\mathrm{B} \pi \mathrm{y} 1, \mathrm{~B} \pi \mathrm{y} 2, \ldots, \mathrm{B} \pi \mathrm{ym})
\end{aligned}
$$

con $\pi \mathrm{x}, \pi \mathrm{y} \in \Omega \mathrm{m}, \pi \mathrm{xn}$ el n-esimo elemento del orden inducido por la permutación $\pi \mathrm{x}$ y $\pi \mathrm{yn}$ el $\mathrm{n}$ esimo elemento del orden inducido por la permutación $\pi$ y. Sea i el punto de corte, entonces se define $\bowtie i$ de la siguiente forma:

$$
\begin{aligned}
& \bowtie i(X, Y) \mapsto Z((B \pi x 1, B \pi x 2, \ldots, B \pi x m),(B \pi y 1, B \pi y 2, \ldots ., B \pi y m)) \mapsto(B \pi x 1, \ldots, B \pi x i \\
& \mathrm{B} \pi y(1), \ldots, B(\mathrm{~B} \pi(m-1))
\end{aligned}
$$

en donde:

- $\quad B \pi y(1)$ es la primera entrada de $Y$ tal que $B \pi y(1) \in / X i:=\{B \pi x 1, \ldots B \pi x i\}$

- $\quad B \pi y(2)$ es la primera entrada de $Y$ tal que $B \pi y(2) \in / X i \cup\{B \pi y(1)\}$

$-\quad \ldots$

- $\quad \mathrm{B} \pi \mathrm{y}(\mathrm{m}-\mathrm{i})$ es la primera entrada de $\mathrm{Y}$ tal que $\mathrm{B} \pi \mathrm{y}(\mathrm{m}-\mathrm{i}) \in / \mathrm{Xi} \cup\{\mathrm{B} \pi \mathrm{y}(1), \ldots, \mathrm{B} \pi \mathrm{y}(\mathrm{m}-\mathrm{i}-1)\}$

Con el operador de cruzamiento definido de la forma anterior, se considera primero la prioridad de $\mathrm{X}$ hasta el fenotipo i-esimo, y después se considera la prioridad del individuo Y para los bloques restantes. Y dadas por:

Por ejemplo, si los bloques a visitar son $\{$ B1, B5, B19, B25, B33, B32\}, sean las soluciones X,

$$
\begin{aligned}
& \mathrm{X}=[\mathrm{B} 1, \mathrm{~B} 5, \mathrm{~B} 25, \mathrm{~B} 19, \mathrm{~B} 33, \mathrm{~B} 32] \\
& \mathrm{Y}=[\mathrm{B} 33, \mathrm{~B} 19, \mathrm{~B} 1, \mathrm{~B} 25, \mathrm{~B} 32, \mathrm{~B} 5] \\
& \text { y el punto de corte } \mathrm{i}=3 \text { entonces: }
\end{aligned}
$$

$$
\mathrm{Z}=\mathrm{X} \bowtie_{3} \mathrm{Y}=[\mathrm{B} 1, \mathrm{~B} 5, \mathrm{~B} 25, \mathrm{~B} 33, \mathrm{~B} 19, \mathrm{~B} 32]
$$

para el punto de corte $\mathrm{i}=2$ se obtiene:

$$
\mathrm{Z}=\mathrm{X} \bowtie_{2} \mathrm{Y}=[\mathrm{B} 1, \mathrm{~B} 5, \mathrm{~B} 33, \mathrm{~B} 19, \mathrm{~B} 25, \mathrm{~B} 32]
$$

\section{Operador de mutación}

El operador de mutación es una operación unaria sobre el espacio de soluciones, que realiza un cambio en el fenotipo del individuos, es decir realiza un cambio en el orden de recorrido permutando la posición de un par de bloques para una trayectoria dada. 
Sea una orden O, y B1, B2, .., Bm los bloques a visitar; sea X una solución dada por:

$\mathrm{X}:(\mathrm{B} \pi \times 1, \mathrm{~B} \pi \mathrm{x} 2, \ldots, \mathrm{B} \pi \mathrm{xm})$

entonces se define $\propto_{(\mathrm{i}, \mathrm{j})}$, el operador de mutación sobre $\Omega_{\mathrm{m}}$, de la siguiente forma:

$\propto(\mathrm{i}, \mathrm{j})(\mathrm{X}) \mapsto \mathrm{Z}(\mathrm{B} \pi \mathrm{x} 1, \ldots, \mathrm{B} \pi \mathrm{xi}, \ldots, \mathrm{B} \pi \mathrm{xj}, \ldots, \mathrm{B} \pi \mathrm{xm}) \mapsto(\mathrm{B} \pi \mathrm{x} 1, \ldots, \mathrm{B} \pi \mathrm{xj}, \ldots, \mathrm{B} \pi \mathrm{xi}, \ldots$

El par de índices (i,j) se toman de forma arbitraria para cada individuo e iteración del algoritmo genético.

por:

Por ejemplo, si los bloques a visitar son $\left\{\mathrm{B}_{1}, \mathrm{~B}_{5}, \mathrm{~B}_{19}, \mathrm{~B}_{25}, \mathrm{~B}_{33}, \mathrm{~B}_{32}\right\}$, y sea $\mathrm{X}$ una solución dada

$$
\begin{aligned}
& \mathrm{X}=\left[\mathrm{B}_{1}, \mathrm{~B}_{5}, \mathrm{~B}_{25}, \mathrm{~B}_{19}, \mathrm{~B}_{33}, \mathrm{~B}_{32}\right] \\
& \text { y el punto de mutación es }(\mathrm{i}=5, \mathrm{j}=2) \text {, entonces: } \\
& \propto_{(2,5)}(\mathrm{X})=\left[\mathrm{B}_{1}, \mathrm{~B}_{33}, \mathrm{~B}_{25}, \mathrm{~B}_{19}, \mathrm{~B}_{5}, \mathrm{~B}_{32}\right] \\
& \text { para el punto de mutación }(\mathrm{i}=1, \mathrm{j}=6) \text { se tiene: } \\
& \propto_{(1,6)}(\mathrm{X})=\left[\mathrm{B}_{32}, \mathrm{~B}_{5}, \mathrm{~B}_{25}, \mathrm{~B}_{19}, \mathrm{~B}_{33}, \mathrm{~B}_{1}\right]
\end{aligned}
$$

Nótese que $\propto_{(\mathrm{i}, \mathrm{j})}(\mathrm{X})=\propto_{(\mathrm{j}, \mathrm{i})}(\mathrm{X})$ para todo $\mathrm{i}, \mathrm{j}$.

\section{Operador de aptitud}

El operador de aptitud mide la calidad de una solución usando la distancia asociada al recorrido, debido a que cada individuo se codifica como una permutación de los bloques a recorrer para una orden dada, para calcular la distancia se usa la matriz de distancias mínima L, calculada de acuerdo a la figura 7.9. Sea una orden $\mathrm{O}$, y B1, B2, .., Bm los elementos de la partición a visitar; sea X una solución dada por:

$$
\mathrm{X}:(\mathrm{B} \pi \mathrm{x} 1, \mathrm{~B} \pi \mathrm{x} 2, \ldots, \mathrm{B} \pi \mathrm{xm})
$$

entonces se define $\amalg(\mathrm{i}, \mathrm{j})$, el operador de aptitud sobre $\Omega \mathrm{m}$, de la siguiente forma:

$$
\begin{aligned}
& \mathrm{U}(\mathrm{X}) \mapsto \mathrm{v} \in R(\mathrm{~B} \pi \mathrm{x} 1, \ldots, \mathrm{B} \pi \mathrm{xi}, \ldots, \mathrm{B} \pi \mathrm{xm}) \mapsto \sum_{\mathrm{i}<\mathrm{m}} \quad \mathrm{L}(\mathrm{B} \pi \mathrm{xi}, \mathrm{B} \pi \mathrm{xi}+1)+\mathrm{L}(\mathrm{B} 0, \mathrm{~B} \pi \mathrm{x} 1)+ \\
& \mathrm{L}(\mathrm{B} 0, \mathrm{~B} \pi \mathrm{xm})
\end{aligned}
$$

en donde $\mathrm{L}(\mathrm{Bx}, \mathrm{By})$ denota la entrada en la matriz de distancias mínima asociada a los elementos de la partición $\mathrm{Bx}, \mathrm{By}$.

El operador de aptitud es una función real valuada y permite ordenar las soluciones por la distancia inversa de recorrido.

\section{Selección y reinserción}

Inicialmente el algoritmo genético crea una población de $\mathrm{K}$ individuos seleccionados aleatoriamente de $\Omega \mathrm{k}$, el espacio de permutaciones asociada de una orden dada $\mathrm{O}$ y entre cada iteración son seleccionados $\mathrm{r}$ individuos para heredar sus características a la siguiente generación. Idealmente se busca a los mejores individuos, aunque para dar variabilidad a la población se les asigna una probabilidad de selección a cada individuo acorde a su aptitud, método de la ruleta (Wolfgang, Nordin, Keller, \& Francone, 1997), y se toman aleatoriamente los r individuos; con este método de selección se permite mayor oportunidad de selección a los mejores individuos sin dejar de considerar a los peores individuos. 
Los individuos seleccionados heredan sus características mediante el operador de cruzamiento, cada uno de ellos se cruza aleatoriamente con algún individuo de la población total. Usando también esta operación de selección se establece a que parte de la población se aplicara la mutación. De esta forma se crea una parte de los individuos que formaran la siguiente generación.

En este trabajo se establece la cuota de individuos generados por cruza en un $40 \%$ del total de la población de y una cuota de un $20 \%$ para los individuos generados por mutación. Se realiza también la selección del mejor individuo de la generación anterior para pasar a la siguiente generación, a esto se le denomina elitismo.

Como el tamaño de la población entre iteraciones siempre es constante, por lo cual se necesita generar un $40 \%$ de la población de nuevos individuos que de forma aleatoria son tomados de $\Omega \mathrm{k}$. Con esto se permite variabilidad en la población y se evitan los mínimos locales en el problema de optimización.

\section{Resultados}

El algoritmo genético utilizado para la realización de los experimentos planteados en la sección Layout del almacén usa los siguientes parámetros fijos para el algoritmo genético:

- $\quad$ Tamaño de la población: 100 individuos.

- $\quad$ Probabilidad de cruza: $20 \%$.

- $\quad$ Probabilidad de mutación: $15 \%$.

- $\quad$ Reinserción: elitismo, el mejor individuo de la generación anterior se conserva en la nueva generación.

- $\quad$ Número de iteraciones: 1000 iteraciones máximo o detener las iteraciones si en las ultimas 100 generaciones no exista mejora en la solución. rangos:

Cada experimento consiste en una tupla de valores $(\mathrm{h}, \mathrm{v}, \mathrm{i})$ que toman valores en los siguientes

- $\quad \mathrm{h}$ el número de pasillos horizontales, toma valores $3,4,5, \ldots, 10$.

- $\quad$ v el número de pasillos verticales, toma valores $3,4,5, \ldots, 10$

- $\quad$ i denota la cantidad de ítems a considerar en la orden, toma valores $5,10,15, \ldots 50$

Las posibles combinaciones anteriores son 8 × 8 × 10=640; cada posible combinación de pasillos horizontales, verticales y cantidad de ítems a recolectar se probo con 20 escenarios o distribuciones espaciales de ítems en el layout generadas aleatoriamente con una distribución de probabilidad uniforme.

Figura 7.10 Resultados generales de los experimentos realizados para todos los layouts distintos e ítems. R estrategia Return, S estrategia S-shape, GAP estrategia Largest Gap y AG estrategia algoritmo genético

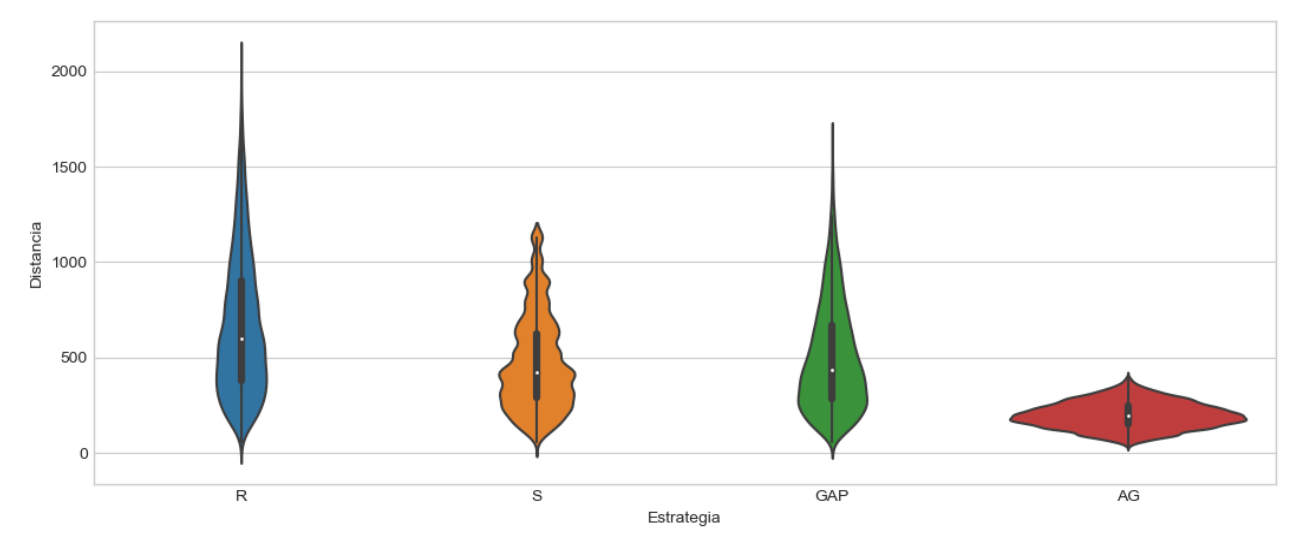


En cada uno de los escenarios generados se comparo el rendimiento de los algoritmos Return, Sshape, Largest Gap y el algoritmo genético obteniéndose los resultados mostrados en la Figura 7.10, observe la distribución de densidad capturada en los gráficos de violín. En promedio el algoritmo genético obtiene mejores resultados en comparación a las demás estrategias y obtiene menor variabilidad en el rango de valores para las rutas calculadas, lo cual indica que el algoritmo genético es mas consistente en la longitud de las rutas.

Figura 7.11 Experimento con 5 ítems en layouts de prueba para estrategias Return (R), S-Shape(S), Largest Gap (GAP) y Algoritmo Genético (AG)

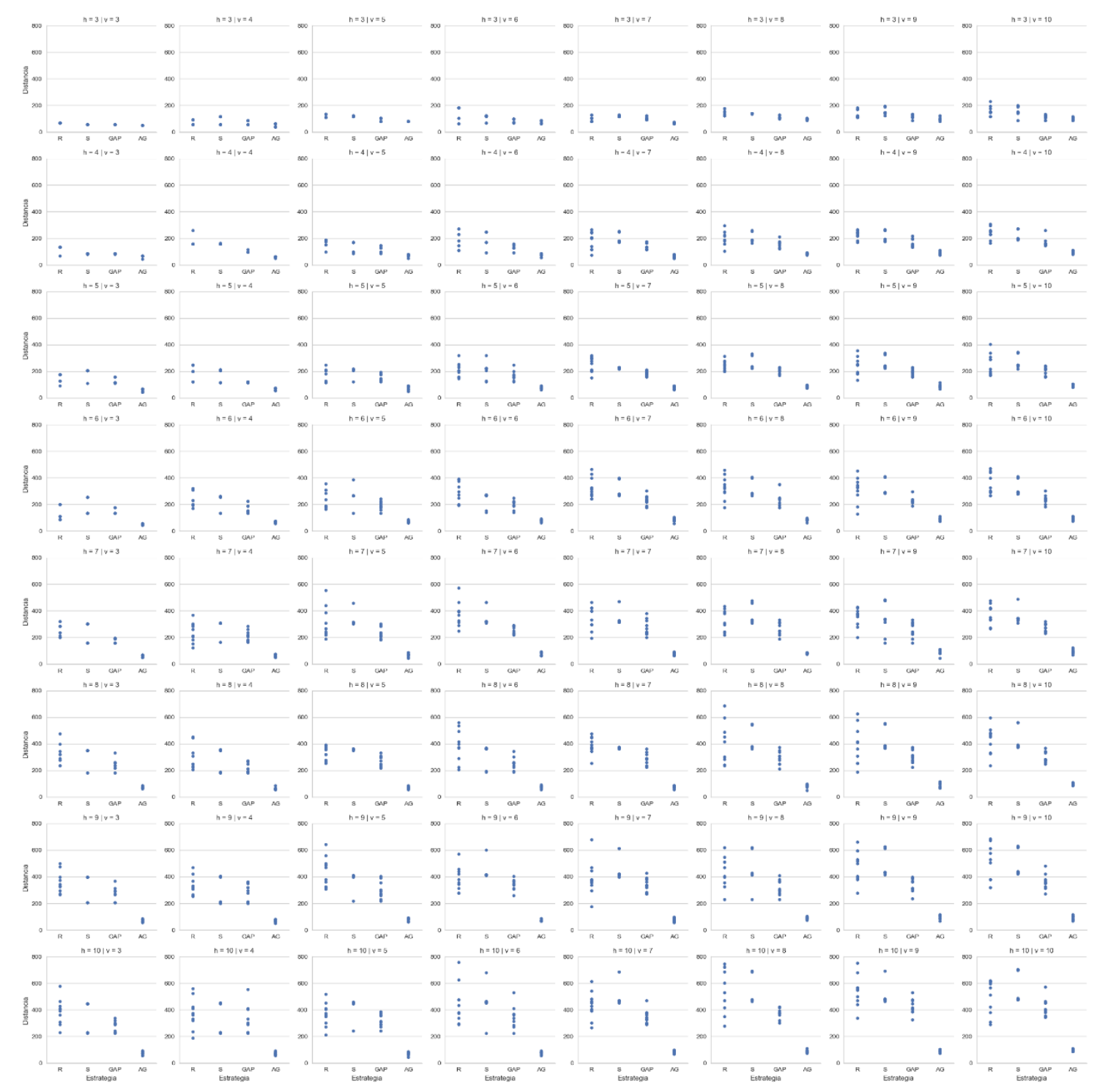

En la Figura 7.11 se muestra el conjunto de pruebas aplicadas para 5 ítems en los layouts considerados para la comparativa. Para el layout $3 \times 3,3$ pasillos horizontales y 3 verticales los algoritmos tienen un rendimiento similar, incrementando los pasillos verticales se empieza a notar diferencias de performance en los algoritmos. Un resultado interesante se obtiene al observar el primer renglón y la primer columna, que corresponden a layouts de almacén tipo $3 \times \mathrm{V} \mathrm{y} \mathrm{H}$ x 3 , el comportamiento no es simétrico. 
Figura 7.12 Experimento con 25 ítems en layouts de prueba para estrategias Return (R), S-Shape(S), Largest Gap (GAP) y Algoritmo Genético (AG)

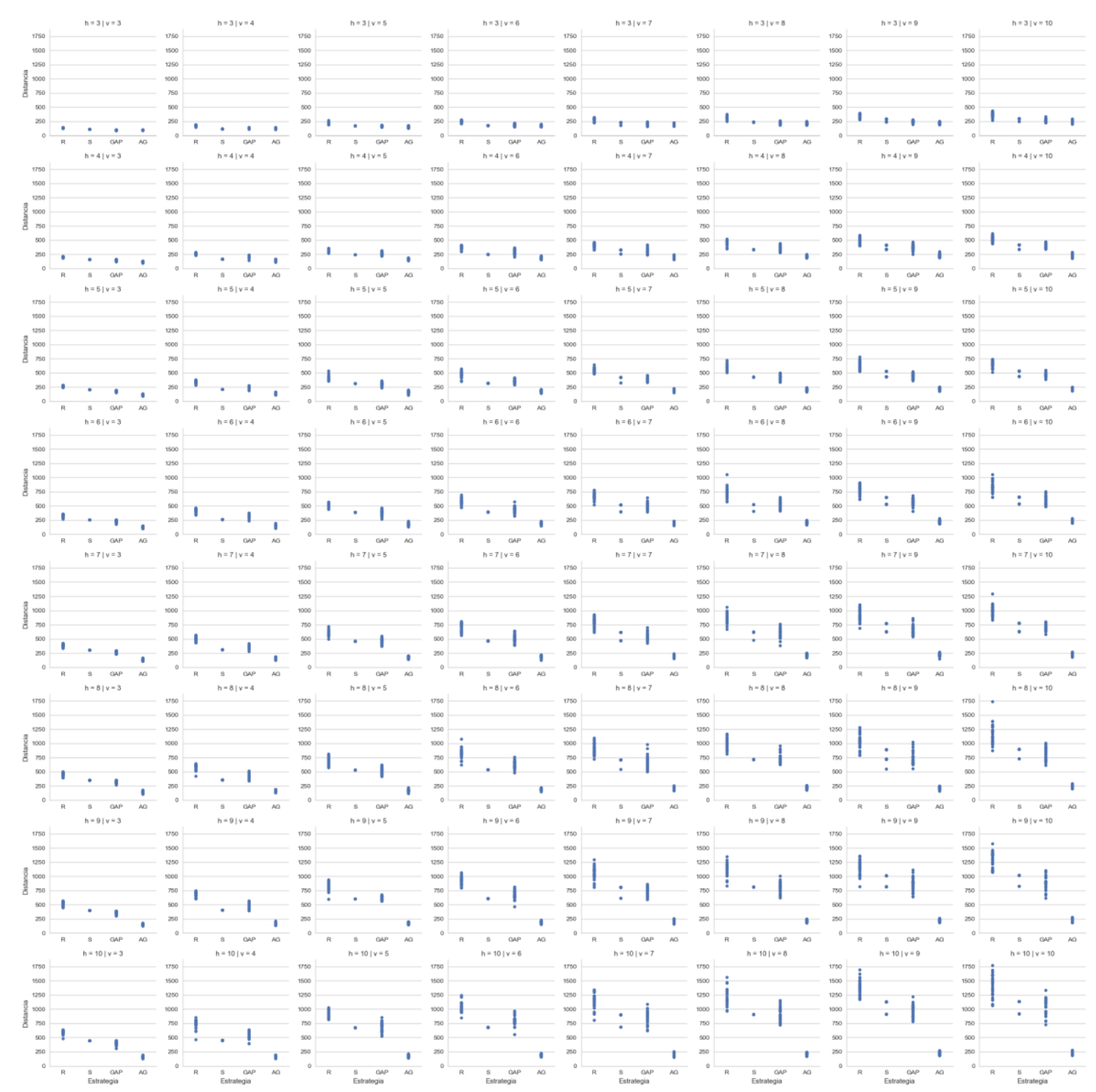

En la figura 7.12 se ilustra el diagrama de dispersión de distancia recorrida para 25 ítems y los distintos layouts de almacén considerados en el experimento; mostrando un comportamiento similar en el primer renglón versus la primer columna, aunque el rango de valores tomado en la primer columna es un poco más amplio. Para el caso de 25 ítems por orden se observa sobre layouts de almacén cuadrados un crecimiento en tiempos de recorrido creciente, pero conservando el performance relativo entre los distintos algoritmos. 
Figura 7.13 Experimento con 50 items en layouts de prueba para estrategias Return (R), S-Shape(S), Largest Gap (GAP) y Algoritmo Genético (AG)

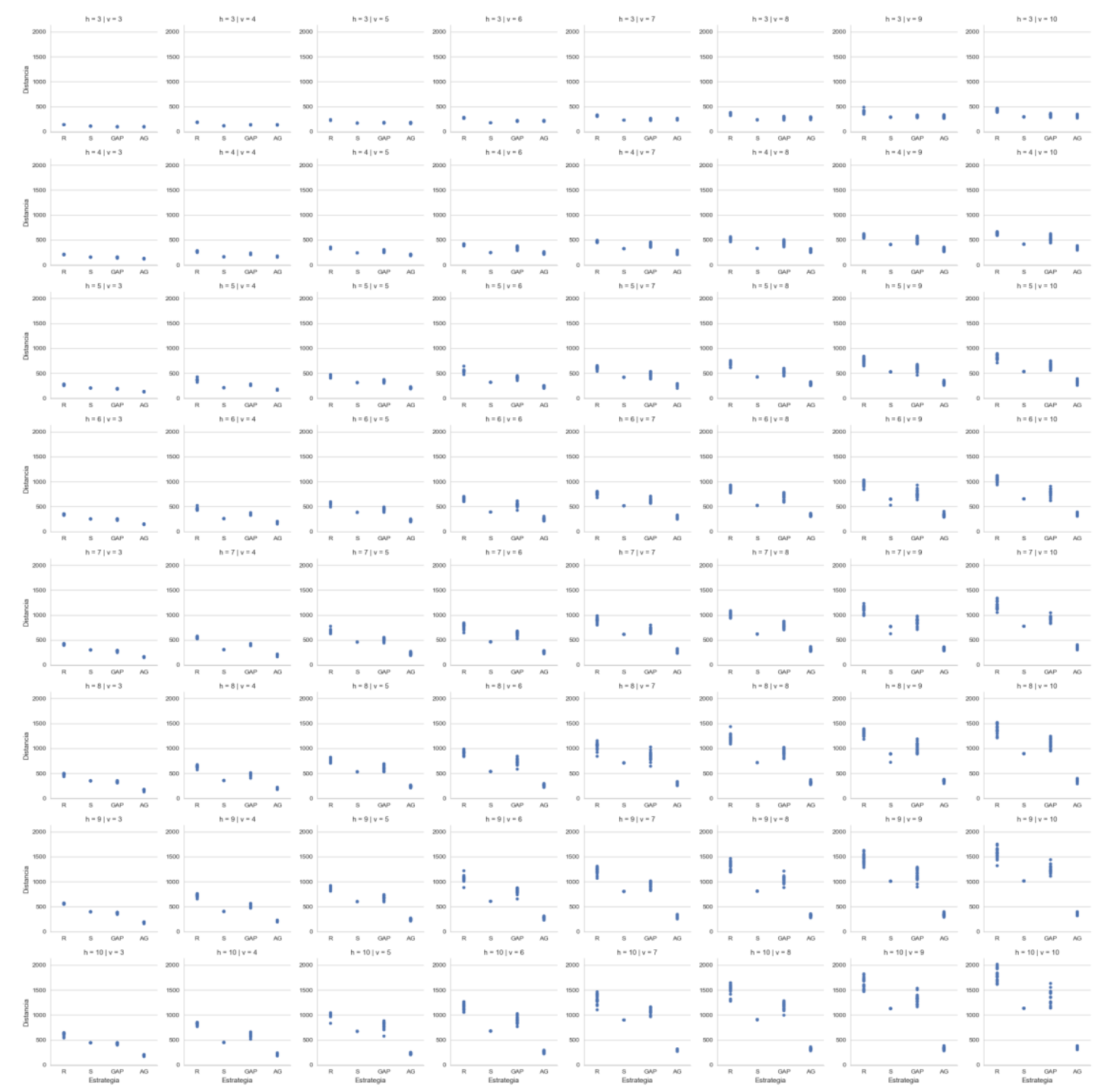

La figura 7.13 ilustra el diagrama de dispersión de distancias recorridas para la simulación realizada con 50 ítems. Comparativamente el primer renglón versus la primer columna en el arreglo de resultados son similares, se conserva el orden en el rendimiento de los algoritmos evaluados; sin embargo la primer columna tiene un recorrido en distancia ligeramente mayor consistente con la distribución de la morfología del layout de los almacenes, estructuras más largas que anchas.

\section{Conclusiones}

Uno de los factores preponderantes en el problema de picking o recolección de ítems para una orden dada en un mínimo de tiempo está dada por el recorrido realizado. La elección de recorrido depende de la estrategia utilizada algunas básicas pero intuitivas y otras eficientes, pero poco prácticas debido al tiempo de computo requerido que dificultan su uso; por lo cual es importante proponer métodos que ayuden a encontrar mejores rutas.

El uso del particionamiento del layout permite reducir la complejidad del problema combinatorial y transformar el número de ítems a recoger a sitios por visitar; esta transformación permite reducir la dimensión del espacio de búsqueda y por ende el tiempo de exploración. La morfología del particionamiento se encuentra inspirado en la política de largest gap que determina cuando es conveniente realizar un recorrido completo del pasillo o solo una parte y dejando el resto para una segunda visita del pasillo. 
Representar la ruta usando únicamente los elementos de la partición del layout reduce la dimensión del espacio de trabajo y simplifica la representación de la trayectoria permitiendo aplicar el principio de optimalidad de Bellman para realizar la búsqueda de la trayectoria de longitud mínima. Para obtener la sucesión completa de sitios a recorrer se presenta un algoritmo que permite decidir la trayectoria mínima entre elementos de la partición.

La aplicación de los algoritmos genéticos usando como modelo de individuo los inducidos por el particionamiento reduce el crecimiento combinatorial del problema y lo hace tratable. Los operadores de cruza y mutación definidos permiten crear nuevos individuos, que heredan características de los padres, que son solución al problema planteado y que tienden a mejorar su calidad.

Los resultados experimentales muestran que, en almacenes grandes, las heurísticas clásicas no consiguen buen rendimiento comparado con el método propuesto usando algoritmos genéticos y presentan mayor dispersión en el rango, por otro lado, se observa que para pocos ítems en general resultan conveniente los almacenes con mas pasillos verticales que horizontales. Para ordenes en las cuales se tienen muchos ítems, 50 en el experimento, es similar el performance entre almacenes largos y anchos; donde un almacén largo es aquel que tiene muchos pasillos horizontales y pocos verticales, en contraparte un almacén ancho es aquel que tiene más pasillos verticales que horizontales.

\section{Referencias}

Aho, A., Ullman, J., \& Hopcroft, J. (1983). Data Structures and Algorithms. Boston, MAUnited States: Addison-Wesley Longman Publishing Co., Inc.

Bartholdi, J., \& Hackman, S. (2014). WAREHOUSE \& DISTRIBUTION SCIENCE Release 0.94. Atlanta, GA USA: The Supply Chain and Logistics InstituteSchool of Industrial and Systems EngineeringGeorgia Institute of Technology.

Bellman, R. (1957). Dynamic Programming. Princeton, NJ, United States: Princeton University Press.

Bozer, Y., \& Kile, J. (2008). Order batching in walk-and-pick order picking systems. International Journal of Production Research, 1887-1909.

Davis, L. (1985). Applying adaptive algorithms to epistatic domains. Proceedings of the 9th international joint conference on Artificial intelligence, IJCAI'85 (págs. 162-164). San Francisco, CA, USA: Morgan Kaufmann Publishers Inc.

Garey, M., \& Jhonson, D. (1979). Computers and Intractability: A Guide to the Theory of NPCompleteness. New York, NY United States: W. H. Freeman.

Henn, S., Koch, S., \& Wäscher, G. (2012). Order Batching in Order Picking Warehouses: A Survey of Solution Approaches. Warehousing in the Global Supply Chain: Advanced Models, Tools and Applications for Storage Systems.

Holland, J. (1992). Adaptation in Natural and Artificial Systems: An Introductory Analysis with Applications to Biology, Control and Artificial Intelligence. Cambridge, MA, United States: MIT Press.

Jinxiang, G., Goetschalckx, M., \& McGinnis, L. (2009). Research on warehouse design and performance evaluation: Acomprehensive review. European Journal of Operational Research, 539-549.

Korte, B., \& Vygen, J. (2010). Combinatorial Optimization: Theory and Algorithms. Germany: Springer.

Nojhan. (3 de April de 2007). Evolutionary algorithm. Recuperado el April de 2020, de Wikimedia Commons: https://commons.wikimedia.org/wiki/File:Evolutionary_algorithm.svg

Roodbergen, K. J., Iris, V., \& Taylor, G. (2015). Simultaneous determination of warehouse layout and control policies. International Journal of Production Research, 3306-3326. 
Scholz, A., Schubert, D., \& Wäsher, G. (2017). Order picking with multiple pickers and due dates Simultaneous solution of Order Batching, Batch Assignment and Sequencing, and Picker Routing Problems. European Journal of Operational Research.

Valle, C., Beasley, J., \& da Cunha, A. (2016). Modelling and Solving the Joint Order Batching and Picker Routing Problem in Inventories. Combinatorial Optimization. ISCO 2016. Lecture Notes in Computer Science, 81-97.

Wolfgang, B., Nordin, P., Keller, R., \& Francone, F. (1997). Genetic Programming - An Introduction. San Francisco, CA: Morgan Kaufmann. 